\title{
In-vitro Strain and Modulus Measurements in Porcine Cervical Lymph Nodes
}

\author{
Queeny Wing-Han Yuen, Yong-Ping Zheng*, Yan-Ping Huang, Jun-Feng He, James Chung-Wai \\ Cheung and Michael Ying
}

\begin{abstract}
Department of Health Technology and Informatics, The Hong Kong Polytechnic University, Kowloon, Hong Kong SAR, China
\end{abstract}

\begin{abstract}
Cervical lymph nodes are common sites of metastatic involvement in head and neck cancers. These lymph nodes are superficially located and palpation is a common practice for assessing nodal hardness and staging cancer which is, however, too subjective and with limited accuracy. In this study, the mechanical properties of pig lymph node tissues were investigated using ultrasound elastography and indentation test. Lymph nodes were excised from fresh pork pieces and embedded in an agar-gelatin phantom for strain imaging by elastography. A strain ratio reflecting the strain contrast of lymph node over agar-gelatin phantom was used to assess the elasticity of the lymph node. A cutting device was then custom-designed to slice the phantom into uniform slices for indentation test. The measurements revealed that there were significant differences in both the strain ratio and Young's modulus between the peripheral and middle regions of the lymph nodes (both $p<0.05$ ); however, the results appeared contradictory. Correlation between the results of the two measurements (modulus ratio vs. inversed strain ratio) showed their association was moderate for both the peripheral and middle regions $\left(R^{2}=0.437\right.$ and 0.424 respectively). As the tests were only performed on normal lymph nodes, comparison in stiffness between healthy and abnormal lymph nodes could not be made. Future studies should be conducted to quantify the stiffness change in abnormal lymph nodes.
\end{abstract}

Keywords: Ultrasound, elastography, indentation, lymph node, soft tissue, strain image, young's modulus.

\section{INTRODUCTION}

Metastases to the lymphatic system are common in most cancers [1]. Cervical lymph nodes are common sites of metastatic involvement in head and neck cancers [2]. Moreover, in the head and neck region, malignant lymphoma is also common in cervical lymph nodes [3]. Although ultrasound assessment of cervical lymph nodes based on the nodal morphology and vasculature has been well established [4], palpation of cervical lymph nodes is still common in clinical practice, particularly when they are large and firm [5]. However, palpation to gauge nodal hardness is considered inaccurate and carries substantial risks of misdiagnosis [6]. Ultrasound elastography, a term first coined by Ophir et al. [7], is a method for imaging tissue strains in response to compression. It has been shown to be a promising technique in the applications of palpable organs such as the breast [8-11], liver [12-15] and thyroid [16, 17].

Information on stiffness of lymph nodes in any species is limited. To date, there is only one study that reported the stiffness of resected lymph nodes [18]. In that study, a tactile sensor was placed on intact human lymph nodes to measure the change of its resonant frequency, which could be used to obtain the tissue stiffness through a calibration process. It was found that metastatic nodes were stiffer than benign

\footnotetext{
*Address correspondence to this author at the Department of Health Technology and Informatics, The Hong Kong Polytechnic University; Tel: 852 27667664; Fax: 852 23624365; E-mail: ypzheng@ieee.org
}

ones, and suggested that stiffness alone was accurate in diagnosing lymph node metastases, ex situ. More recently, using the elastography technique, in vivo studies have been conducted on skin surface for superficial cervical nodes [1922] or endoscopically for deep cervical, mediastinal and abdominal nodes [23-27], to study nodal stiffness. Most of these studies utilized the EUB8500 (Hitachi Medical Corp., Tokyo, Japan) system; and strain images of tissue produced by freehand compression were color-coded according to its magnitude relative to the surrounding tissues and transparently superimposed on the conventional grey-scale ultrasound image. Elastographic color range of lymph nodes was reported to be between green and dark blue, which represents intermediate to hard tissue regions, depending on the degree of malignancy [21, 22, 27]. Although operator dependence and perception error when visually grading subtle color differences can be overcome by computerized quantitative analysis [27], few studies have provided absolute values of the stiffness of lymph nodes [18]. Therefore, studies that further the current understanding of the relationship between strain and stiffness measurements (such as Young's modulus) are required. Furthermore, biomechanical testing could extend our limited understanding of nodal tissue stiffness and such data were suggested being helpful in improving and validating elastography results $[20,27]$.

Therefore, the aim of the present study was to investigate the stiffness of lymph node tissues using a pig model. In common with that of wildlife species such as dolphins, elephants and rhinoceroses, the lymph node of domestic pig is structurally inverted; with tissue characteristic of the medulla 
on the periphery and tissues characteristic of the cortex tissue in center $[28,29]$. Indentation as a frequently used technique to study the biomechanical properties of biological tissues was adopted as a small indenter tip allows determination of any differences in tissue stiffness among selected regions. It is expected that information obtained from this study could help understand better the lymph node from an anatomical aspect. Also, it may help to improve the interpretation of elastograms in the assessment of lymphadenopathy.

\section{MATERIALS AND METHODS}

\section{Sample Preparation}

Sizable irregular pieces of pork meat, cut from the posterior head and neck regions, were acquired from a local wet market in early mornings. All the specimens reached our lab within six hours after pigs were sacrificed. B-mode ultrasonography was conducted on the meat specimens to identify and locate suitable lymph nodes for investigation. The selected lymph nodes were resected from the surrounding tissues and measured and weighed with a digital calliper and an electronic scale respectively. Volume was determined by a water displacement method. To prevent dehydration, the selected lymph nodes were wrapped in gauze soaked in physiological saline and stored at $4^{\circ} \mathrm{C}$ in a sealed container until further use.

\section{Phantom Fabrication}

On the same day as resection, the lymph nodes were embedded in phantom of agar-gelatin blocks. Fabrication of the tissue mimicking agar-gelatin background in this study was based on previous reports [30-32]. Briefly, gelatin (SigmaAldrich Inc., MO, USA) and agar (Sigma-Aldrich Inc., MO, USA) were mixed with water and the mixtures were separately heated in water baths until a solution state was reached. The solutions were allowed to cool down to about $50{ }^{\circ} \mathrm{C}$ and then mixed in volume proportions of $60 \%$ gelatin and $40 \%$ agar. The mixture was constantly stirred and at approximately $36^{\circ} \mathrm{C}$, the mixture was poured carefully into an acrylic mould $(10 \mathrm{~cm} \times 10 \mathrm{~cm} \times 13 \mathrm{~cm}$, Fig. 1) containing a lymph node. The lymph node was suspended using a thin thread to occupy a central position, with the long axis being orthogonal to the direction of slicing in preparation of specimens for the indentation test. In order for an easy slicing, two $2 \mathrm{~mm}$ longitudinal slits, $2 \mathrm{~mm}$ apart, was cut on opposite walls to guide a sharp knife (Fig. 1). Prior to pouring the agar-gelatin mixture into the mould, the slits were temporarily sealed using thin plastic sheets. The gel mixture was placed in a refrigerator $\left(4^{\circ} \mathrm{C}\right)$ overnight for solidification.

\section{Elastography}

Ultrasound examination was conducted on the following day. The phantom was first taken out from the refrigerator and left in the air for some time to reach room temperature, and then scanning was carried out with the block remaining inside the mould. B-mode ultrasonography and elastography were performed using the Hitachi EUB-8500 system (Hitachi Medical Cor., Tokyo, Japan) in conjunction with a 6-14 $\mathrm{MHz}$ linear transducer (4 $\mathrm{cm}$ footprint length). The orientation of the transducer for imaging was orthogonal to the long axis of each lymph node. The plane with the largest transverse diameter of the embedded lymph node was identified

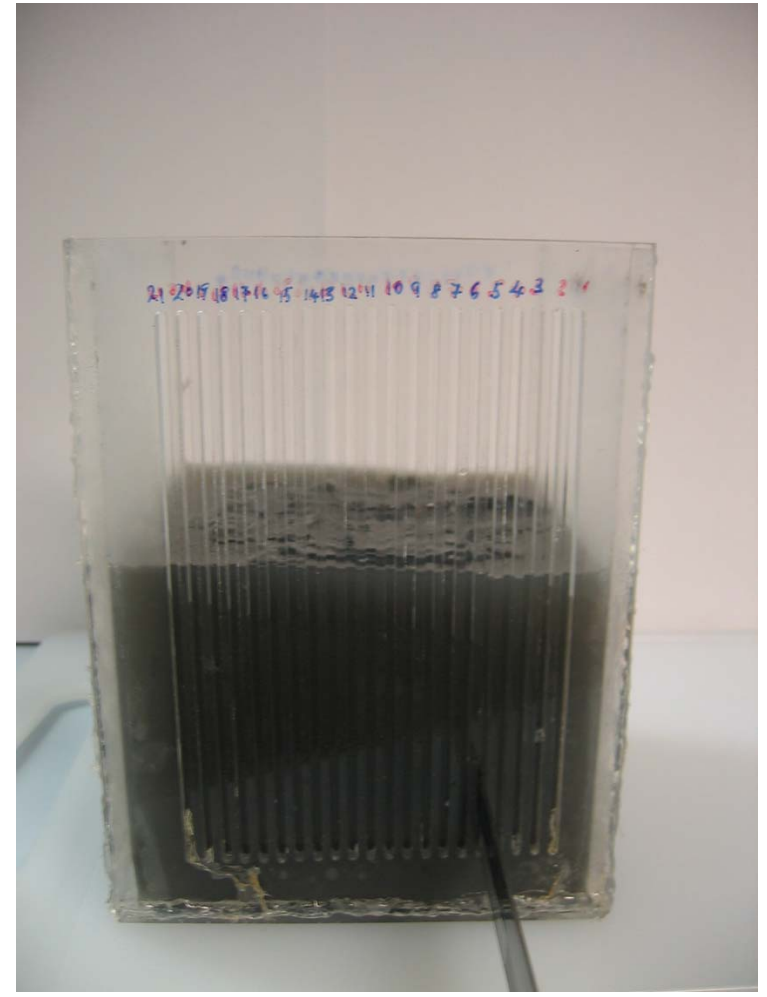

Fig. (1). Custom-made mould with slits on opposite sides for guiding the knife during slicing.

and elastography was initially conducted on this plane followed by the rest of the lymph node to gain an overall understanding of the elasticity of entire structure. Elasticity images were obtained through strategic selection of the regions of interest so that they included an appropriate proportion of the surrounding phantom background, and appropriate compressions could be performed using freehand operation in the vertical direction. For a given image (or frame) obtained from one compression, multiple small areas were selected within the region of interest for the calculation of strain ratios, using the system's inbuilt function. The strain ratio was defined as the ratio of two user-selected regions and in this study it meant the ratio of strain in the lymphatic tissue region over that in the agar-gelatin phantom region. The absolute strain value was not used directly because it was easily affected by the local stress level and boundary conditions, which were hard to be controlled in elastographic measurement. Sampling areas for strain ratios included regions within the lymph node (peripheral and middle) and in the phantom background material.

\section{Indentation Test}

After ultrasonography examination, the plastic sheets that covered the side slits of the mould were removed. Under ultrasound guidance, a sharp knife was placed through the slits (in the same orientation as imaging) for slicing. The slice with largest cross-section of the lymph node was used for indentation testing (Fig. 2a). Slice specimens were covered with physiological saline soaked gauze while waiting for testing to avoid dehydration.

The experimental setup is shown in Fig. 2b. Indentation was conducted at room temperature $\left(23-24^{\circ} \mathrm{C}\right)$. The indenter used was flat headed and $3 \mathrm{~mm}$ in diameter. It was coupled 
a)

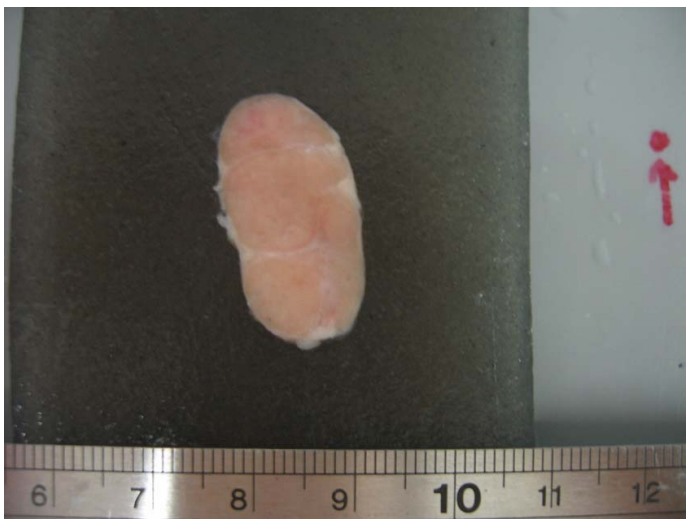

b)

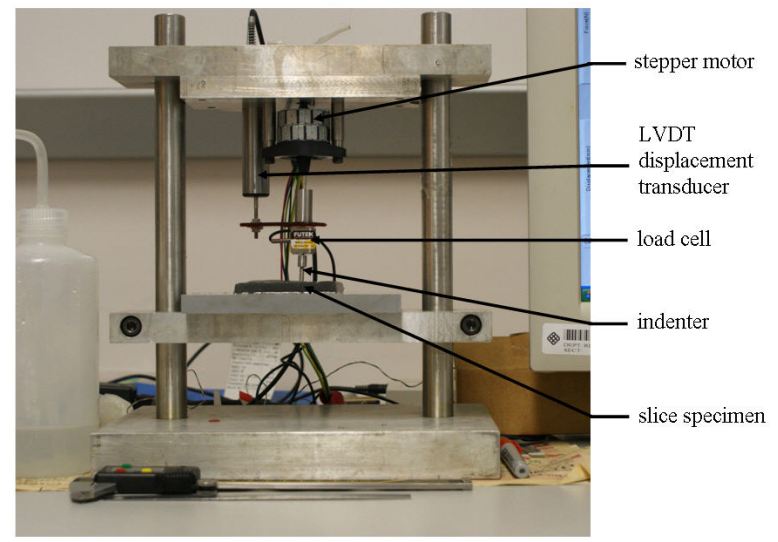

Fig. (2). a) A sliced sample of lymph node for indentation test and b) the experimental set up for the indentation test.

to a $1 \mathrm{~N}$ load cell (LSB200, Futek Advanced Sensor Technology Inc., Irvine, CA, USA) and driven by a linear stepper motor with a precision of $0.03 \mathrm{~mm}$ (UBL23, Saia-Burgess Inc., OH, USA) which was in turn coupled to a linear variable differential transformer (LVDT) displacement transducer (DC15, Salartron Metrology Ltd., Leicester, UK) by a custom-made device. The entire system was computer controlled through the user-interface of software developed inhouse using LabWindows / CVI 8.5 (National Instruments Co., TX, USA).

The thickness of each slice was measured by a digital calliper, and caution was taken to keep a minimal compression on the specimen during the measurement. Slice specimens were placed on a horizontal platform wetted with thin layer of physiological saline that provided lubrication and prevented the specimen from dehydration. The platform was adjusted vertically so that the starting position of the indenter was slightly above the slice specimen. Indentation tests, each consisted of 5 cycles of loading and unloading, were performed at a indenter speed of approximately $0.5 \mathrm{~mm} / \mathrm{s}$. The maximum indentation depth was set to be about $20 \%$ of the initial thickness of the slice. An indentation model proposed by Hayes et al. [33] on articular cartilage was adopted in this study and the Young's modulus of the tissue at the indentation site was extracted as follows [33, 34]:

$$
E=\frac{1-v^{2}}{2 a \kappa(v, a / h)} \cdot \frac{P}{w}
$$

where $E$ is the Young's modulus of the tissue, $a$ is the indenter radius, $v$ the Poisson's ratio of the tissue, $h$ the initial thickness, $\kappa$ a correction factor related to $v$ and the aspect ratio $a / h$ ( $\kappa$ table can be found in the study of Hayes et al. [33]), and $P / w$ the relationship between force and indentation depth. A Matlab (MathWorks Inc., Natick, MA, USA) program was written for computing the Young's modulus based on the force/indentation data. $v$ was assumed to be a constant value of 0.45 , indicating a near incompressibility of the tested tissue, which is a common assumption and similar values of Poisson's ratio have been reported in previous studies [35,36]. A pre-load of $0.03 \mathrm{~N}$ and a maximum deformation of $10 \%$ were set for extracting the Young's modulus. From pilot studies, it was found the preconditioning effect was not so serious in the lymph node tissues and it nearly disappeared after two cycles of indentations (Fig. 3). Therefore, to reduce the potential effect of preconditioning, data of the first two cycles were neglected. Each point underwent two repeated tests with a one-minute interval. For a given slice, the number of points tested depended on the cross-sectional size of the lymph node and multiple points were tested within its peripheral and middle regions. To reduce the effect of background phantom material in comparison of stiffness to the strain ratio, a modulus ratio, i.e., the ratio of Young's modulus of the lymph node to

a)

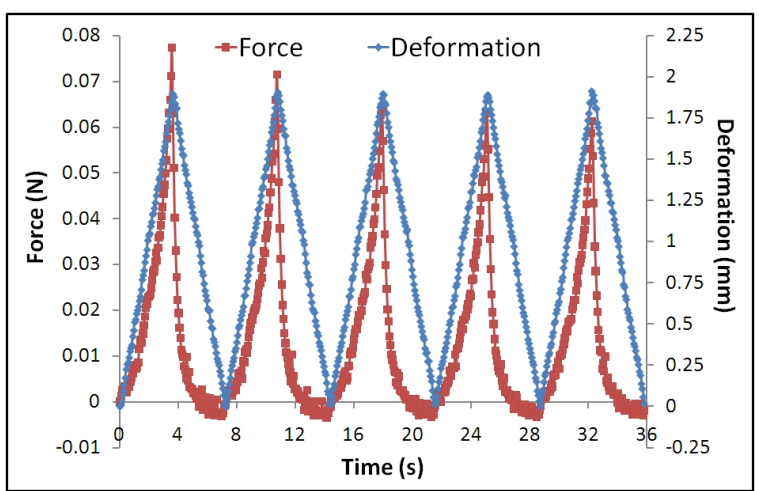

b)

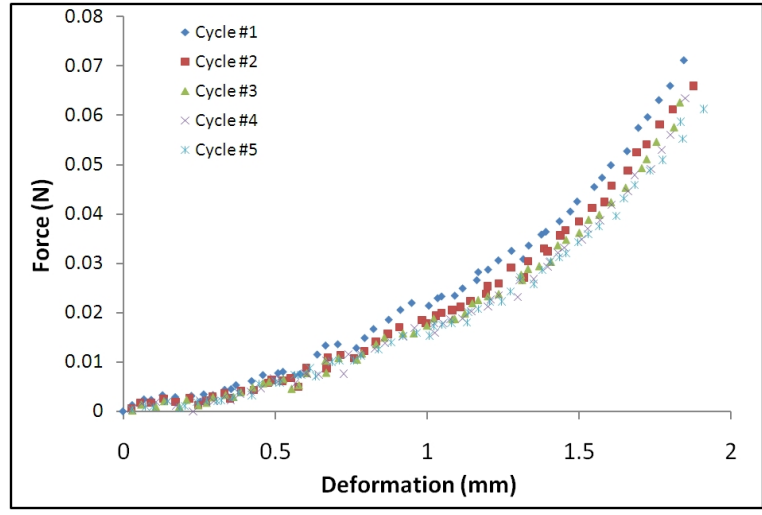

Fig. (3). a) A typical indentation curve of force and deformation showing five cycles of loading and unloading with time and (b) the corresponding relationship of force and deformation in the loading phase for the five cycles. Different cycles were separated using different markers. 
that of the phantom material, was also used for the analysis of results.

\section{Statistical Analysis}

Paired- $t$ testes were used to determine any difference in stiffness between overall lymph node tissue and background phantom and between peripheral and middle regions within the lymph nodes. Pearson product-moment correlation coefficients, $R$, were obtained to examine the strength of associations between the strain and modulus measurements. A level of $P<0.05$ was considered to be significant. All statistical analysis was done using SPSS 14.0 for Windows (SPSS Inc., IL, USA).

\section{Results}

A total of 22 lymph nodes, obtained from 11 pigs (two for each pig), were examined. The size of the nodes selected for investigation ranged from $1.3 \mathrm{~cm}^{3}$ to $10.1 \mathrm{~cm}^{3}$ (Mean: 4.7 $\pm 2.4 \mathrm{~cm}^{3}$ ) and $1.1 \mathrm{~g}$ to $9.2 \mathrm{~g}$ (Mean: $4.2 \pm 2.3 \mathrm{~g}$ ) in volume and mass, respectively.

\section{Gross Anatomical Description}

Lymph nodes were palpated from the skin surface to roughly identify their locations within the piece of flesh. On excision, as the structures were well-circumscribed by a capsule, it was easy to isolate them from the surrounding connective fat and muscles. Macroscopic examination revealed that the shape of each lymph node was irregular and the surface of the capsule was uneven (Fig. 4). Further dissection confirmed that a node may be composed of several smaller nodelets, as described by previous researchers [28, 29]. In cross-section, although the divisions can be clear, individual nodelets were difficult to separate without causing damage. Therefore, no further attempt was made to isolate each nodelet and the whole lymph node was embedded into the agar-gelatin block for testing.

\section{B-mode and Elastography Observation}

For majority of the lymph nodes, bright lines that divided the structure into smaller sections were readily seen and small echolucent areas surrounded by echogenic borders were also common features. A typical B-mode image of the lymph node is shown in Fig. (5).

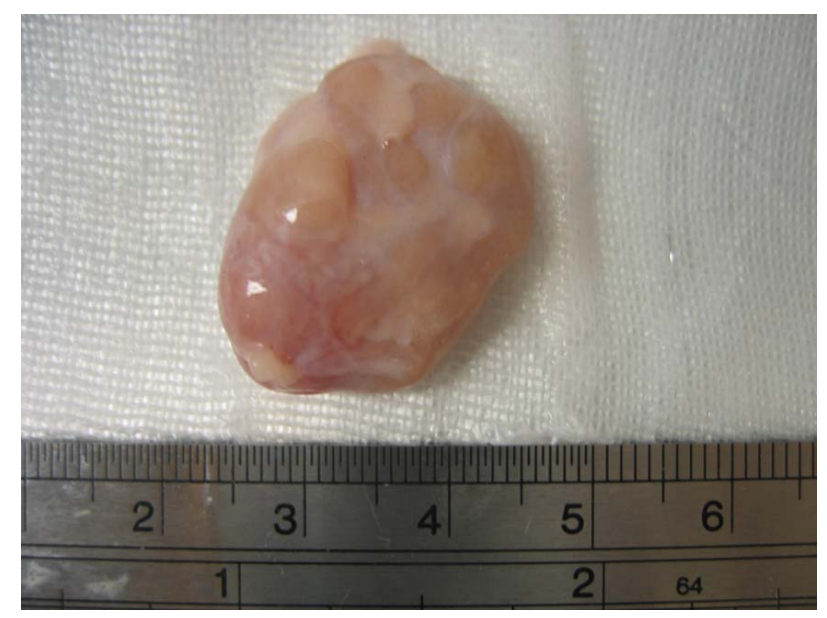

Fig. (4). A resected lymph node showing the uneven surface.
A typical elastogram of the lymph node consisted of a blue belt of non-uniform thickness around the periphery of the structure and different shades of green, with speckles or streaks of yellow and red towards the centre. These features were observed to correspond well with the echogenic and echolucent areas in the B-mode images (Figs. 6a-b). And the two kinds of images combined provided in greater detail the internal architecture of a lymph node. Also, for some lymph nodes, elastography revealed more distinct contrast between the lymph node and surrounding material than B-mode imaging (Fig. 7).

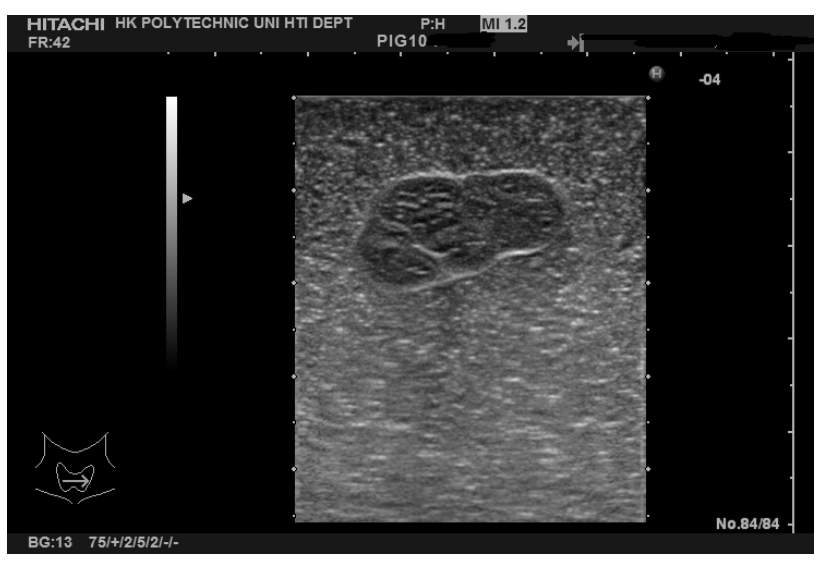

Fig. (5). B-mode image of a lymph node with apparent internal divisions and echolucent areas.

a)

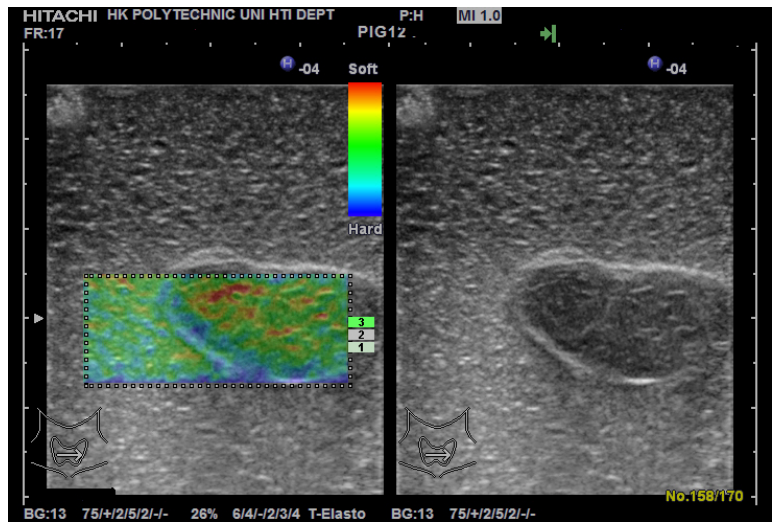

b)

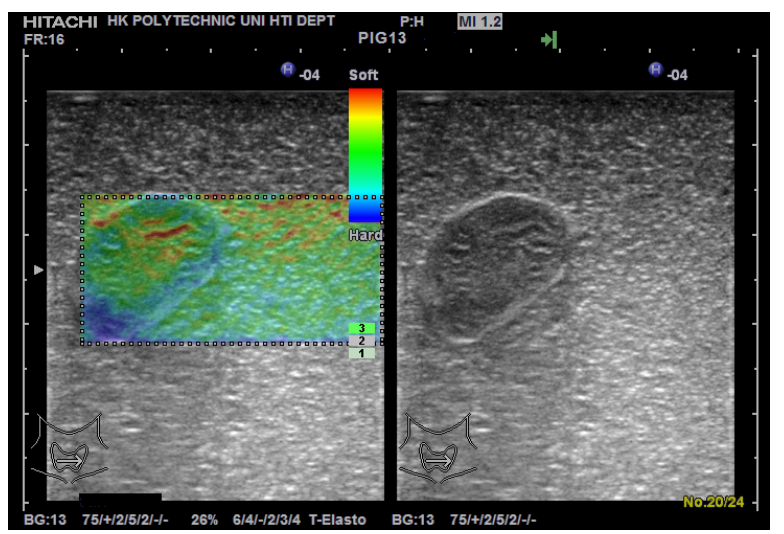

Fig. (6). Visual correspondence of nodal internal patterns between elastograms and B-mode images: a) cross-section along the long axis and $\mathbf{b}$ ) along the short axis. 


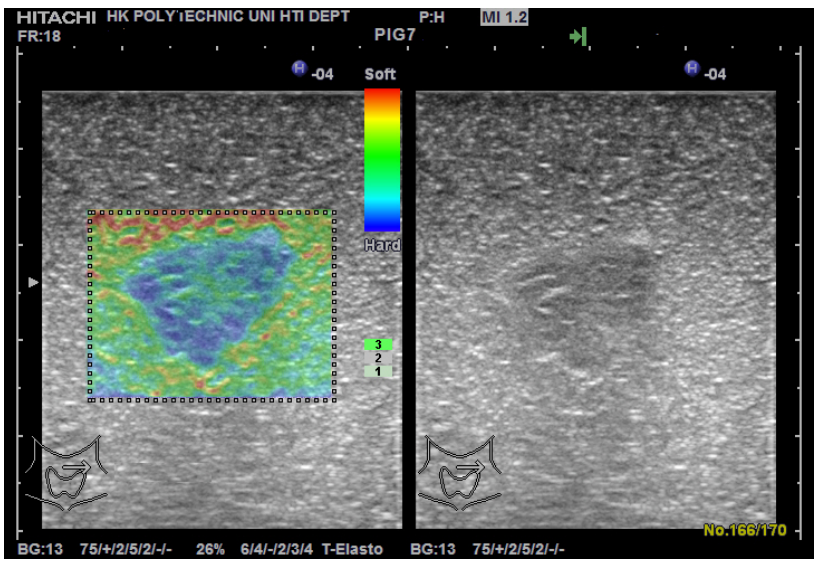

Fig. (7). An elastogram showing a clearer nodal border with the background phantom materials in comparison with the corresponding B-mode image.

\section{Strain Ratios}

The mean strain ratio between the peripheral region of the lymph nodes and phantom material was $0.61 \pm 0.32$ (mean $\pm \mathrm{SD}$ ), while that between the central region and the phantom material was $0.93 \pm 0.33$ (Table 1). Statistical analysis revealed a significant difference $(P<0.05)$, indicating that the peripheral tissue was stiffer and therefore exhibited less strain when compression was applied. This finding was in alignment with the typical elastogram pattern as described above.

No significant association was found between the lymph node size (volume and mass) and the strain ratio. Degrees of association between the two parameters varied from weak to fair $(R=0.2-0.5$, all $P>0.05)$.

\section{Indentation Test}

One slice of specimen for each lymph node with the largest cross-sectional area was obtained from the agar-gelatin block and underwent the indentation test. A total of 457 points in 21 lymph node slices was tested (one sample was not sliced successfully).

The result of indentation test showed that there was a significant difference $(P<0.05)$ between the Young's modulus of the surrounding background material $(11.78 \pm$ $2.80 \mathrm{kPa})$ and that of the overall lymph node tissue $(26.06 \pm$ $6.03 \mathrm{kPa}$ ). Both the peripheral and middle regions of the lymph node tissue were generally stiffer than the background material by more than two folds (Table 1). Furthermore, it

Table 1. Results of Elastography and Indentation Tests (mean \pm SD). The Ratios were Obtained with the Reference to the Readings of the Surrounding Phantoms

\begin{tabular}{|c|c|c|}
\hline & Peripheral Region & Middle Region \\
\hline \hline Elastography strain ratio & $0.61 \pm 0.32 *$ & $0.93 \pm 0.33$ \\
\hline $\begin{array}{c}\text { Indentation Young's modulus } \\
(\mathrm{kPa})\end{array}$ & $25.39 \pm 6.14 *$ & $26.73 \pm 5.64$ \\
\hline Indentation modulus ratio & $2.21 \pm 0.60$ & $2.33 \pm 0.51$ \\
\hline
\end{tabular}

*indicates a significant difference $(P<0.05)$. was found that the Young's modulus of the peripheral region $(25.39 \pm 6.14 \mathrm{kPa})$ was slightly but significantly $(P<0.05)$ smaller than that of the middle region $(26.73 \pm 5.64 \mathrm{kPa})$. For the modulus ratio, there was no significant difference between the peripheral and middle regions of the lymph node $(P>0.05)$ (Table $\mathbf{1})$.

\section{Comparison Between Strain and Indentation Data}

To investigate the association between the strain and modulus measurements, correlations were made between the Young's modulus/modulus ratio and the inversed strain ratio for both the peripheral and middle regions (Fig. 8a-d) of the lymph nodes. There was no significant correlation between the Young's modulus and the inversed strain ratio in both regions of the lymph node $(P>0.05)$ (Fig. 8a-b). However, moderately significant correlations between the modulus ratio and the inversed strain ratio were found for both the peripheral and middle regions $\left(R^{2}=0.437\right.$ and 0.424 , respectively, $P<0.05$, Fig. 8c-d).

\section{DISCUSSION}

Metastatic lymph nodes tend to be hard, whereas benign nodes are usually soft. Clinically, palpation is still commonly performed by ear, nose and throat (ENT) surgeons and oncologists for the assessment of cervical lymphadenopathy, which is however too subjective. In this study, with the aim to provide a quantitative assessment of the lymph nodes stiffness, the tissue stiffness of the pig lymph nodes was measured using two methods: elastography of the whole lymph node for strain measurement and indentation of sliced lymph node specimen for modulus determination. The relationship between strain and modulus distribution has been studied in some detail by Srinivasan et al. [37, 38]. In those studies, phantoms, with sponge inclusions of various degree of stiffness, as well as cut slices of various tissue types such as bovine muscle, canine prostate, etc. were investigated. Different from those studies, in the present research, the whole lymph nodes were embedded into the agar-gelatin blocks for elastography and only sliced specimen was tested by indentation.

For both the peripheral and middle regions, no significant correlation was found between the Young's modulus and the inversed strain ratio, which might be because the inversed strain ratio is a reflection of stiffness contrast of the lymph node to the phantom material while the Young's modulus is representative of absolute stiffness of the lymphatic tissues. Therefore, it was more appropriate to use the modulus ratio to compare with the inversed strain ratio. When the modulus ratio was used, only moderate correlation between the results by the two methods was observed, as the test results could be affected by several factors such as quality of the elastography images, interfacial bonding, boundary conditions, tissue geometry and dimension [38-40]. Although elastography by freehand compression appeared to be simple and easy to conduct, operation of the EUB 8500 system and application of the appropriate compression with the transducer did require a period of training to ensure acquisition of acceptable quality images for accurate determination of strain ratios. To achieve this and avoid inter-operator variation, the operator received at least one month's training and carried out all the elastography examinations. During slicing, the interfacial bonding between the background and lymph node appeared 
a)

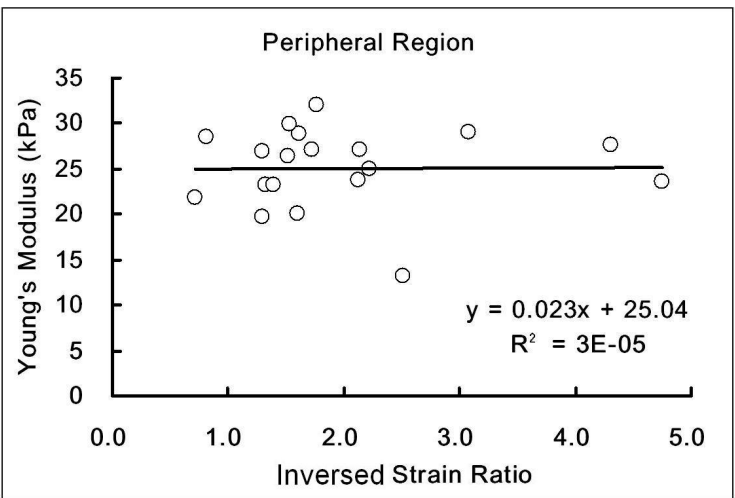

b)

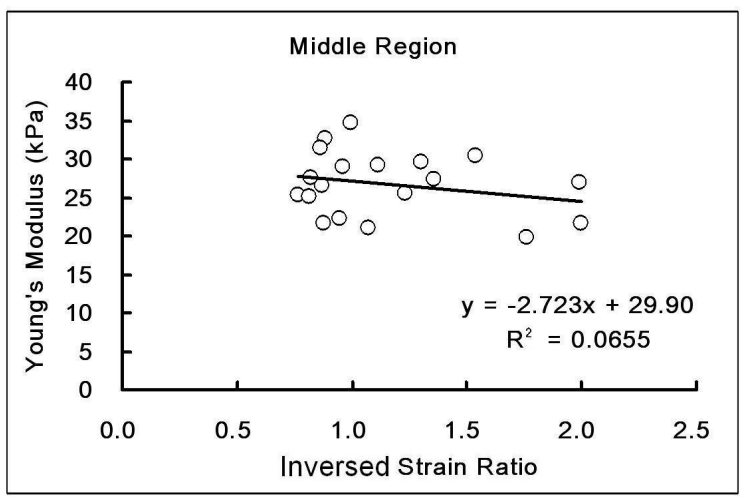

c)

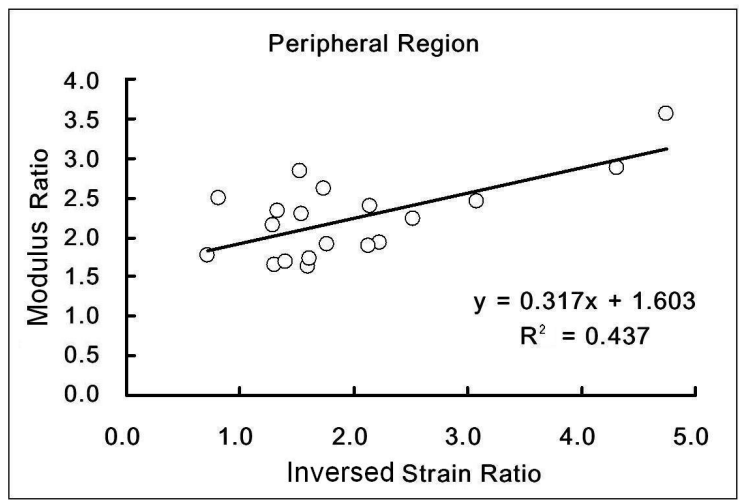

d)

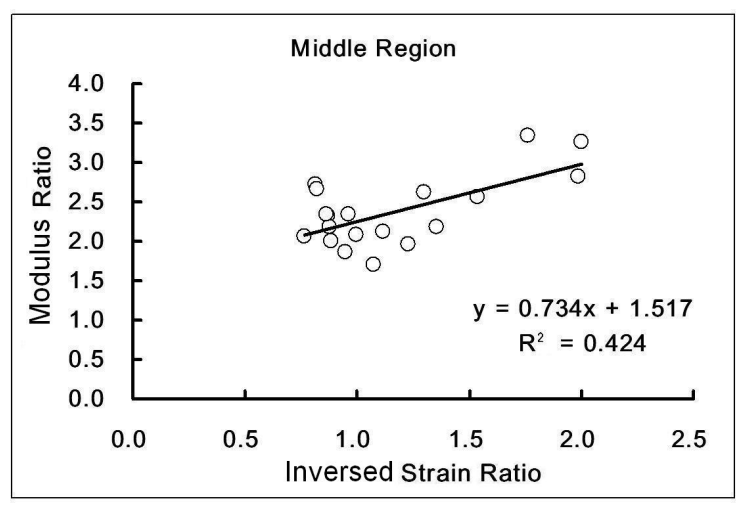

Fig. (8). Correlations between Young's modulus/modulus ratio and inversed strain ratio for the peripheral and the middle regions of the lymph nodes. (a) and (b) are related to the results of Young's modulus. (c) and (d) are related to the results of modulus ratio. to be secure; however, the degree of bonding was not objectively tested. A recent study reported that the assumption of firm background-inclusion bonding could induce a varying degree of error in the estimation of strain measurements depending on the selection of the region of interest within the inclusion [40]. Such finding bears important clinical relevance as benign and malignant tumours would differ in their bonding with the surrounding tissues [41]. The pig lymph nodes investigated in the present study had distinct smooth boundaries, and thus was easily separated from the surrounding connective tissues. But when embedded into the agargelatin blocks, some connective tissues were left attached to the lymph nodes to promote or increase surface area for bonding with the phantom material. After elastography, dissection or cutting of a lymph node for indentation impaired the function of the capsule, which is primarily to provide mechanical strength and structural integrity [42]. An in-vitro study showed that the stiffness of bovine liver differed by a factor of 2 to 3 when the capsule was removed [43]. The strain result of this study showed that the stiffness of the middle region of the lymph nodes was similar to that of the background material and the peripheral region (smaller strain) was harder than the middle region. However, the indentation test revealed the central region (larger Young's modulus) was stiffer than the outside region. The discrepancy might be explained by the breakdown of the capsule barrier; and the difference of direction of compression in strain imaging and indentation test (orthogonal to each other) could be another reason. In elastographic measurement, the compression was parallel with the slice surface used in the indentation test while it was perpendicular to the slice in the indentation test. The potentially anisotropic mechanical properties of lymph node warrant further investigation. Previous nanoindentation studies on phantoms and soft tissues reported that a spatial resolution of $2 \mathrm{~mm}$ generated modulus images corresponding well with strain images or elastograms $[37,38]$. However, due to the absence of an automated stage controller to locate the indentation points on a sliced specimen, a spatial resolution on the order of at least $3 \mathrm{~mm}$ was not fully realized in this study. The insufficient spatial resolution may also have caused the disagreement between the results of the two tests. Furthermore, the strain measurements were performed on different sites of the lymph node to give an overall understanding of the strain distribution throughout the structure. However, they did not correspond exactly to the plane where modulus measurements were made.

Repeatability of the indentation system was found to be of satisfactory level. Prior to this study, a separate repeatability study was conducted on 12 silicone samples with different stiffness. An intra-class correlation coefficient (ICC) of 0.99 was found, indicating a repeatability of $99 \%$. Also, a repeatability coefficient (in accordance with Bland and Altman [44]) of $10.7 \mathrm{kPa}$ was ascertained. With reference to this coefficient, the differences in Young's modulus between the surrounding background material $(11.78 \pm 2.80 \mathrm{kPa})$ and lymph node tissues $(26.06 \pm 6.03 \mathrm{kPa})$ could be attributed to an inherent difference in stiffness.

Due to equipment limitation, the sliced specimens were quite thick, i.e. $>7 \mathrm{~mm}$. Sample thickness is an important factor to consider, especially for pigs, as their lymph nodes may contain multiple nodelets, thus the internal tissue can be 
highly heterogeneous. Thinner slices were preferred as they tend to be more homogenous throughout the tissue depth. In a previous study, thin tissue slices with a thickness of 2- 3 $\mathrm{mm}$ and a small cross-section of $3 \times 2 \mathrm{~cm}^{2}$ were attained by freehand cutting with a sharp knife [38]. In this study, the cutting method was developed to produce slice specimens with uniform thickness across a larger cross-sectional area $(8-10 \mathrm{~cm}$ in width and length). Our pretest trial suggested that thicker slices were of better quality for the indentation test in our study compared to thinner slices.

The tests were only conducted on normal lymph nodes as they were cut from pigs which were sold in the market and supposed to be healthy. During our test, we found that some nodes appeared to be congested and haemorrhagic. It was not certain whether this was caused by post-mortal changes or trauma, or it was an indication of abnormality or a disease. As the number of such abnormal samples was small, they were excluded from our study. Therefore, comparison between healthy and abnormal lymph nodes could not be made in our current study. It would be interesting to explore the strain and modulus changes in abnormal lymph nodes in future studies.

In summary, this study tried to investigate the stiffness of pig lymph node by measuring strain and Young's modulus using elastography and indentation test, respectively. Both the tests revealed significant differences in the parameters between peripheral and middle regions of the lymph node and the results by the two measurements were found to be moderately correlated. Future studies should be conducted to explore whether the two methods could be used to observe the stiffness change in abnormal lymph nodes.

\section{ACKNOWLEDGEMENTS}

This research was partially funded by grants from the Hong Kong Innovation and Technology Commission (GHP/061/05), the Research Grant Council (PolyU5332/07E, PolyU5339/08E) and the Hong Kong Polytechnic University (J-BB69). The authors wish to thank fellow colleagues for their technical advice and assistance in this investigation.

\section{REFERENCES}

[1] S. Das, and M. Skobe, "Lymphatic vessel activation in cancer," Ann. NY Acad. Sci., vol. 1131, pp. 235-41, 2008.

[2] R. J. Baatenburg de Jong, R. J. Rongen, J. S. Lameris, M. Harthoorn, C. D. A. Verwoerd, and P. Knegt, "Metastatic neck disease-palpation vs ultrasound examination," Arch. Otolaryngol. Head Neck Surg., vol. 115, pp. 689-90, Jun 1989.

[3] C. A. DePena, P. Van Tassel, and Y. Y. Lee, "Lymphoma of the head and neck," Radiol. Clin. N. Am., vol. 28, pp. 723-43, Jul 1990.

[4] A. Ahuja, and M. Ying, "Sonography of neck lymph nodes. Part II: Abnormal lymph nodes," Clin. Radiol., vol. 58, pp. 359-66, May 2003.

[5] P. Delaere, "Clinical and endoscopic examination of the head and neck," in Head and Neck Cancer Imaging, R. Hermans, Ed. Heidelberg: Springer, pp. 17-29, 2006.

[6] J. A. Castelijns, and M. W. M. van den Brekel, "Neck nodal disease," in Head and Neck Cancer Imaging, R. Hermans, Ed. Heidelberg: Springer, pp. 293-309, 2006.

[7] J. Ophir, I. Cespedes, H. Ponnekanti, Y. Yazdi, and X. Li, "Elastography - a quantitative method for imaging the elasticity of biological tissues," Ultrason. Imaging, vol. 13, pp. 111-134, Apr 1991.

[8] T. Shiina, and E. Ueno, "In vivo breast examination by real-time freehand elasticity imaging system," in Research and Development in Breast Ultrasound, E. Ueno, T. Shiina, M. Kuboto, and K. Sawai, Eds. Tokyo: Springer, pp. 7-15, 2005.

[9] A. Itoh, E. Ueno, E. Tohno, H. Kamma, H. Takahashi, T. Shiina, M. Yamakawa, T. Matsumura, "Breast disease: clinical application of US elastography for diagnosis," J. Radiol., vol. 239, pp. 341-50, May 2006.

[10] H. Zhi, B. Ou, B. M. Luo, X. Feng, Y. L. Wen, and H. Y. Yang, "Comparison of ultrasound elastography, mammography, and sonography in the diagnosis of solid breast lesions," J. Ultrason. Med., vol. 26, pp. 807-15, Jun 2007.

[11] A. Tardivon, C. El Khoury, F. Thibault, A. Wyler, B. Barreau, and S. Neuenschwander, "Elastography of the breast: a prospective study of 122 lesions," J. Radiol., vol. 88, pp. 657-62, May 2007.

[12] D. C. Rockey, "Noninvasive assessment of liver fibrosis and portal hypertension with transient elastography," Gastroenterology, vol. 134, pp. 8-14, Jan 2008.

[13] L. Castera, J. Vergniol, J. Foucher, B. Le Bail, E. Chanteloup, M. Haaser, M. Darriet, P. Couzigou, V. De Ledinghen, "Prospective comparison of transient elastography, fibrotest, APRI, and liver biopsy for the assessment of fibrosis in chronic hepatitis C," Gastroenterology, vol. 128, pp. 343-50, Feb 2005.

[14] B. Coco, F. Oliveri, A. M. Maina, P. Ciccorossi, R. Sacco, P. Colombatto, F. Bonino, M.R. Brunetto, "Transient elastography: a new surrogate marker of liver fibrosis influenced by major changes of transaminases," J. Viral Hepatitis, vol. 14, pp. 360-9, May 2007.

[15] A. Sagir, A. Erhardt, M. Schmitt, and D. Haussinger, "Transient elastography is unreliable for detection of cirrhosis in patients with acute liver damage," Hepatology, vol. 47, pp. 592-5, Feb 2008.

[16] A. Lyshchik, T. Higashi, R. Asato, S. Tanaka, J. Ito, J. J. Mai, P.B. Claire, F. I. Michael, B. B. Aaron, S. Tsuneo, H. Masahiro, T. Kaori, "Thyroid gland tumor diagnosis at US elastography," Radiology, vol. 237, pp. 202-11, Oct 2005.

[17] U. Bae, M. Dighe, T. Dubinsky, S. Minoshima, V. Shamdasani, and Y. M. Kim, "Ultrasound thyroid elastography using carotid artery pulsation - preliminary study," J. Ultrason Med., vol. 26, pp. 797-805, Jun 2007.

[18] K. Miyaji, A. Furuse, J. Nakajima, T. Kohno, T. Ohtsuka, K. Yagyu, T. Oka, S. Omata, "The stiffness of lymph nodes containing lung carcinoma metastases - a new diagnostic parameter measured by a tactile sensor," Cancer, vol. 80, pp. 1920-5, Nov 1997.

[19] M. K. Furukawa, A. Kubota, H. Hanamura, Y. Fujita, and M. Furukawa, "Diagnosis of cervical lymph node metastasis of head and neck squamous cell carcinoma - usefulness of power Doppler ultrasonography and elastography," Medix Supplement, pp. 20-3, 2003.

[20] A. Lyshchik, T. Higashi, R. Asato, S. Tanaka, J. Ito, M. Hiraoka, M.F. Insana, A.B. Brill, T. Saga, K. Togashi, "Cervical lymph node metastases: diagnosis at sonoelastography - initial experience," Radiology, vol. 243, pp. 258-67, Apr 2007.

[21] F. Alam, K. Naito, J. Horiguchi, H. Fukuda, T. Tachikake, and K. Ito, "Accuracy of sonographic elastography in the differential diagnosis of enlarged cervical lymph nodes: comparison with conventional B-mode sonography," Am. J. Roentgenol., vol. 191, pp. 604-10, Aug 2008.

[22] S. Aoyagi, K. Izumi, H. Hata, H. Kawasaki, and H. Shimizu, "Usefulness of real-time tissue elastography for detecting lymphnode metastases in squamous cell carcinoma," Clin. Exp. Dermatol., vol. 34, pp. E744-7, Dec 2009.

[23] M. Giovannini, L. C. Hookey, E. Bories, C. Pesenti, G. Monges, and J. R. Delpero, "Endoscopic ultrasound elastography: the first step towards virtual biopsy? Preliminary results in 49 patients," Endoscopy, vol. 38, pp. 344-8, Apr 2006.

[24] M. Giovannini, B. Thomas, B. Erwan, P. Christian, C. Fabrice, E. Benjamin, M. Genevieve, A. Paolo, D. Pierre, Y. Robert, S. Walter, S. Hanz, S. Carl, D. Christoph, E. Pierre, L. Jean-Luc van, D. Jacques, V. Peter, and S. Adrian, "Endoscopic ultrasound elastography for evaluation of lymph nodes and pancreatic masses: a multicenter study," World J. Gastroenterol., vol. 15, pp. 1587-93, Apr 2009.

[25] A. Saftoiu, and P. Vilmann, "Endoscopic ultrasound elastography a new imaging technique for the visualization of tissue elasticity distribution," J. Gastrointestin. Liver Dis., vol. 15, pp. 161-5, Jan 2006.

[26] J. Janssen, C. F. Dietrich, U. Will, and L. Greiner, "Endosonographic elastography in the diagnosis of mediastinal lymph nodes," Endoscopy, vol. 39, pp. 952-7, Nov 2007. 
[27] A. Saftoiu, P. Vilmann, T. Ciurea, G. L. Popescu, A. Iordache, H. Hassan, F. Gorunescu, S. Iordache, "Dynamic analysis of EUS used for the differentiation of benign and malignant lymph nodes," Gastrointest. Endoscopy, vol. 66, pp. 291-300, Aug 2007.

[28] R. M. Binns, "Organization of the lymphoreticular system and lyphocyte markers in the pig," Vet. Immunol. Immunopathol., vol. 3,pp. 95-146, 1982.

[29] M. A. Bowes, and A. J. Kenny, "Endopeptidase-24.11 in pig lymph nodes - purification and immunocytochemical localization in reticular cells," Biochem. J., vol. 236, pp. 801-10, Jun 1986.

[30] C. L. de Korte, E. I. Cespedes, A. F. W. van der Steen, B. Norder, and K. T. te Nijenhuis, "Elastic and acoustic properties of vessel mimicking material for elasticity imaging," Ultrason. Imaging, vol. 19, pp. 112-26, Apr 1997.

[31] T. J. Hall, M. Bilgen, M. F. Insana, and T. A. Krouskop, "Phantom materials for elastography," IEEE Trans. Ultrason. Ferroelectr. Freq. Control, vol. 44, pp. 1355-65, Nov 1997.

[32] E. L. Madsen, M. A. Hobson, H. R. Shi, T. Varghese, and G. R. Frank, "Tissue-mimicking agar/gelatin materials for use in heterogeneous elastography phantoms," Phys. Med. Biol., vol. 50, pp. 5597-618, Dec 2005.

[33] W. C. Hayes, G. Herrmann, L. F. Mockros, and L. M. Keer, "A mathematical analysis for indentation tests of articular cartilage," $J$. Biomech., vol. 5, pp. 541-51, 1972.

[34] Y. P. Zheng, A. F. T. Mak, and B. Lue, "Objective assessment of limb tissue elasticity: development of a manual indentation procedure," J. Rehabil. Res. Dev., vol. 36, pp. 71-85, Apr 1999.

[35] Y. P. Zheng, S. F. Leung, and A. F. T. Mak, "Assessment of neck tissue fibrosis using an ultrasound palpation system: a feasibility study," Med. Biol. Eng. Comput., vol. 38, pp. 497-502, Sep 2000.
[36] T. R. Tilleman, M. M. Tilleman, and M. H. A. Neumann, "The elastic properties of cancerous skin: Poisson's ratio and Young's modulus," Isr. Med. Assoc. J., vol. 6, pp. 753-755, Dec 2004.

[37] S. Srinivasan, T. Krouskop, and J. Ophir, "A quantitative comparison of modulus images obtained using nanoindentation with strain elastograms," Ultrasound Med. Biol., vol. 30, pp. 899918, Jul 2004.

[38] S. Srinivasan, T. Krouskop, and J. Ophir, "Comparing elastographic strain images with modulus images obtained using nanoindentation: preliminary results using phantoms and tissue samples," Ultrasound Med. Biol., vol. 30, pp. 329-43, Mar 2004.

[39] Y. C. Fung, Biomechanics: Mechanical Properties of Living Tissues. New York: Springer, 1993.

[40] A. Thitaikumar, and J. Ophir, "Effect of lesion boundary conditions on axial strain elastograms: a parametric study," Ultrasound Med. Biol., vol. 33, pp. 1463-7, Sep 2007.

[41] E. Ueno, "Classification and diagnostic criteria in breast echography," Jpn. J. Med. Ultrason., vol. 13, pp. 19-31, 1986.

[42] F. G. Hall, "The functional anatomy of lymph nodes," in Lymph Node Biopsy Interpretation, A. G. Stansfeld and A. J. D'Ardenne, Eds. London: Churchhill Livingstone, pp. 3-28, 1992.

[43] M. Hollenstein, A. Nava, D. Valtorta, J. G. Snedeker, and E. Mazza, "Mechanical characterization of the liver capsule and parenchyma," Lect. Notes Comput. Sci., vol. 4072, pp. 150-8, 2006.

[44] J. M. Bland, and D. G. Altman, "Statistical methods for assessing agreement between two methods of clinical measurement," Lancet, vol. 327, pp. 307-10, Feb 1986.

Received: September 07, 2010

Revised: January 07, 2011

Accepted: January 07, 2011

(C) Yuen et al.; Licensee Bentham Open.

This is an open access article licensed under the terms of the Creative Commons Attribution Non-Commercial License (http://creativecommons.org/ licenses/by-nc/3.0/) which permits unrestricted, non-commercial use, distribution and reproduction in any medium, provided the work is properly cited. 